\title{
SEMICONDUCTOR CONDUCTIVITY
}

\author{
Gulhayo Oybekovna Akbarova \\ Teacher of Andizhan State University, \\ Andizhan, \\ Republic of Uzbekistan
}

Article DOI: https://doi.org/10.36713/epra2867

\begin{abstract}
In given article has been expressed solids, free carriers during their motion continuously experience collisions with atoms and ions of impurities and various defects, as a result, free carriers scatter as well.
\end{abstract}

KEY WORDS: semiconductor, conductivity, source, ion, experience, atoms.

\section{INTRODUCTION}

In solids, free carriers during their motion continuously experience collisions with atoms and ions of impurities and various defects, as a result, free carriers scatter. In the presence of an electric field, uniformly accelerated carrier motion occurs only in the intervals between collisions. After each collision, the free particle begins to accelerate again. During scattering, the energy and direction of motion of the carriers change which is accompanied by the excitation or absorption of phonons. The average velocity of the ordered movement of electrons and holes, directed along the electric field, which is called the drift velocity ( $\mathrm{v}$ other), is proportional to the electric field strength $\mathrm{E}$ :

$$
\text { V other }=\boldsymbol{\mu} \varepsilon_{\text {.(1) }}
$$

The proportionality coefficient between $\boldsymbol{\mu}$ the drift velocity $\mathrm{v}$ other and the electric field $\mathrm{E}$ is called mobility and has the dimension $\mathbf{M}^{2} /(\mathbf{B} \cdot \mathbf{C})$.

\section{METHODS}

Due to the difference in the effective masses of the electrons $\boldsymbol{m}_{\boldsymbol{n}}$ and holes $\boldsymbol{m}_{\boldsymbol{p}}$, their mobilities can vary greatly. So, for example, in silicon, the mobility of electrons is almost three times greater than the mobility of holes. With greater carrier mobility, the speed of semiconductor devices will be higher. The mobility of carriers in semiconductors depends on many factors, the most important of which are temperature, the concentration of impurities, and also the electric field strength. The dependence of mobility on temperature $T$ is determined by the scattering of carriers on the sites of the crystal lattice and on impurity ions. When scattering by impurity ions, the temperature dependence of mobility has the form

$$
\mu_{\text {ион }}=\mu_{0 \text { ион }}\left(\mathrm{T}_{0} / \mathrm{T}\right)^{3 / 2}(2)
$$

then

If scattering by lattice atoms predominates,

$$
\boldsymbol{\mu}_{\text {pew }}=\boldsymbol{\mu}_{0 \text { реш }}\left(\mathrm{T}_{0} / \mathrm{T}\right)^{-a}(3)
$$

\section{RESULTS AND DISCUSSIONS}

$$
\text { In formula } \boldsymbol{\mu}_{\text {ион }}=\boldsymbol{\mu}_{0} \text { ион }\left(\mathrm{T}_{0} / \mathrm{T}\right)^{3 / 2} \text { и } \boldsymbol{\mu}
$$

реш $=\boldsymbol{\mu}_{0}$ реш $\left(\mathbf{T}_{0} / \mathbf{T}\right)^{-\mathrm{a}}$ that $\boldsymbol{T}_{\boldsymbol{\sigma}}-$ some initial (initial) temperatures at which the mobility is 0 . The exponent "a" depends on the material and the type of conductivity; so, for $n$-type silicon ( $\mathrm{p}$-type) $\mathrm{a}=2.42$ $(\mathrm{a}=2.2)$; respectively, for Ga As $\mathrm{a}=1(\mathrm{a}=2.1)$.

When both of the above carrier scattering processes are taken into account, the expression for mobility has the following form:

$$
(1 / \mu)=\left(1 / \mu_{\text {ион }}\right)+\left(1 / \mu_{\text {peш }}\right)(4)
$$

Carrier scattering from thermal vibrations of the semiconductor crystal lattice mainly dominates at relatively high temperatures and low impurity concentrations. At high temperatures, due to an increase in the amplitude of thermal vibrations of lattice atoms, the frequency of collisions of charge carriers with them increases, which leads to a more intense scattering of them. Thus, with increasing temperature, the mobility of the carriers decreases due to their scattering by the thermal vibrations of the lattice. 
Carrier scattering by impurity ions prevails at low temperatures and high impurity concentrations. If the temperature is low, then the thermal velocity of the electron is also small, therefore, when the impurity moves relative to the ion, the electron trajectory changes strongly randomly, which corresponds to larger scattering. With increasing temperature, due to an increase in the speed of electrons, the time of their Coulomb interaction with impurity ions decreases, which leads to less carrier scattering. Thus, upon scattering by impurity ions, the carrier mobility increases with increasing temperature in accordance with the dependence

$$
\boldsymbol{\mu}_{\text {ион }} \sim \mathrm{T}^{3 / 2}
$$

At constant temperature, the resulting mobility of the carriers substantially depends on the impurity concentration. If it is small, then, as noted above, the main role, as in pure semiconductors, is played by scattering by thermal vibrations of the lattice (phonons), while the mobility is of maximum importance. With an increase in the impurity concentration $\mathrm{N}$, scattering by impurity ions begins to dominate, as a result of which the carrier mobility decreases (Fig. 1), where $\boldsymbol{\mu}_{\mathrm{n}}, \boldsymbol{\mu}_{\mathrm{p}}$ - respectively, the mobilities of electrons and holes[1.262]

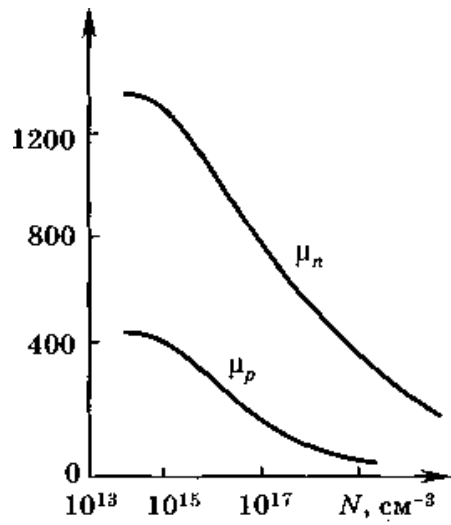

The dependence of mobility on the electric field is ambiguous. In weak electric fields, when the field strength $\mathrm{E}<103 \ldots 104 \mathrm{~V} / \mathrm{cm}$, the drift velocity turns out to be much lower than the thermal velocity of the electrons. In this case, the field does not significantly affect the scattering of carriers, the mobility does not depend on the value of $\mathrm{E}$, and the drift velocity $V$ other, in accordance with the formula $\mathrm{v}$ other $=$, increases linearly with increasing field strength (Fig. 2).В сильных электрических полях $\left(\mathrm{E} \geq 1 \mathbf{1 0}^{4} \ldots \mathbf{1 0}^{5} \mathrm{~B} / \mathrm{sm}\right)$ The carrier drift velocity approaches the average thermal velocity, and the average directed electron motion energy becomes sufficient to increase the amplitude of atomic
Figure 1

vibrations at the nodes of the semiconductor crystal lattice, which leads to an increase in the collision frequency and, accordingly, to intensification of the carrier scattering process.

As a result, with increasing $\mathrm{E}$, the carrier mobility decreases at a constant drift velocity (see Fig. 2) [2.25]. In gallium arsenide, the dependence of mobility is more complicated compared to Si and Ge, which is associated with the features of the conduction band. These features are analyzed in the following chapters when considering processes in some types of optoelectronic devices and lasers, as well as Gunn diodes. 


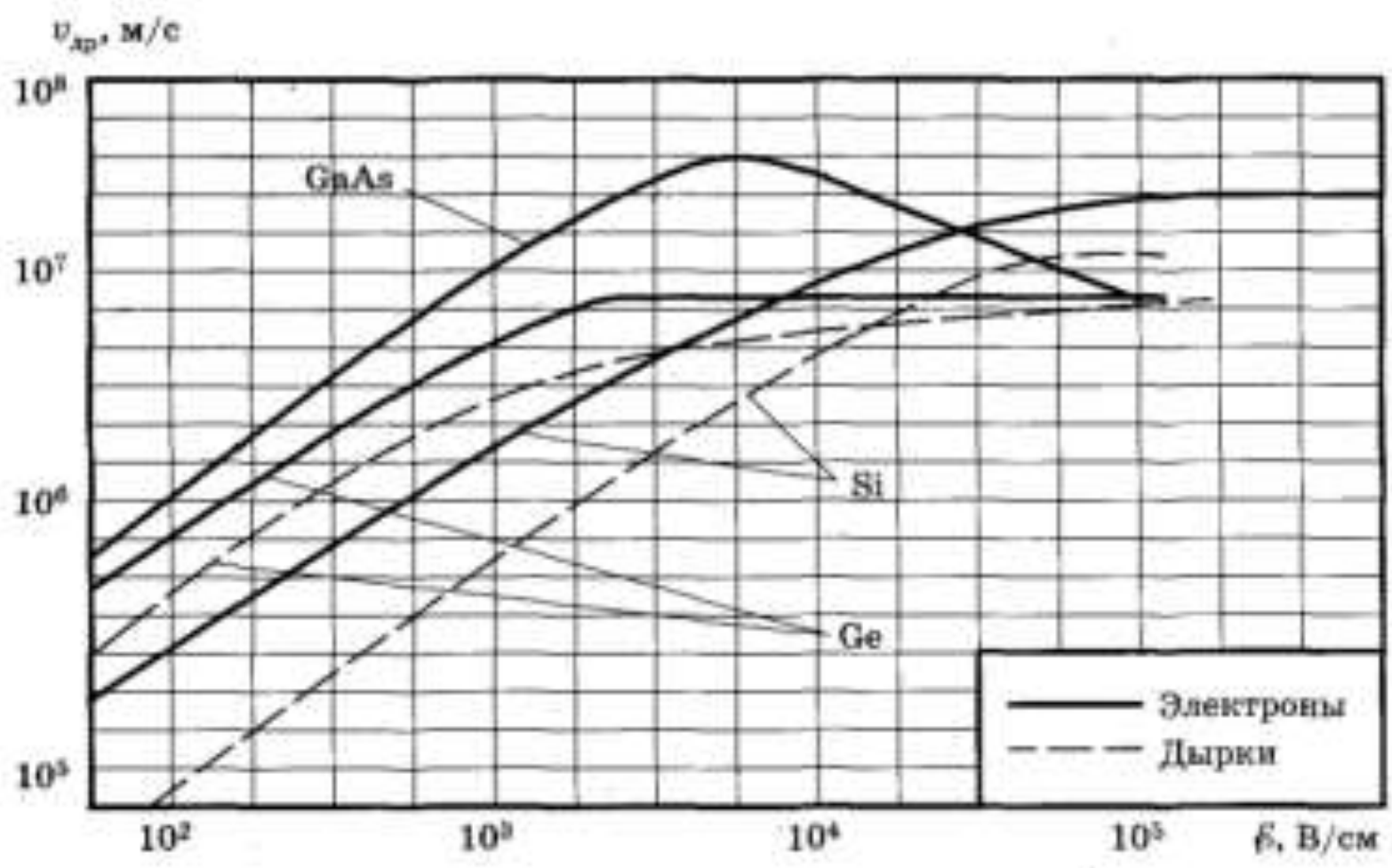

Figure 2.

Knowing the specifics of the behavior of mobility and concentration depending on various physical and electrophysical conditions, one can analyze such an important parameter as the electrical conductivity of semiconductors. According to Ohm's generalized law, the density of the drift current $\mathrm{jdr}$ flowing through the medium, the field strength $\mathrm{E}$ and the electrical conductivity of the medium are related by the ratio $j d r=\mathrm{E}$. The density of the drift current depending on the charge carrier concentration and their drift velocity $v d r$ is $j d r=q N v d r$ where $\mathrm{q}$ is the particle discharge, $\mathrm{N}$ is the concentration of particles of a certain type.

For electrons, $\mathrm{N}=\mathrm{n}$, and for holes, $\mathrm{N}=\mathrm{p}$. If there are charges of both types in a semiconductor, the current density will be determined by the sum of the currents of positive and negative charges, that is,

$$
\mathrm{j}_{\mathrm{Ap}}=\mathrm{q}\left(\mathrm{n} \boldsymbol{v}_{\mathrm{Ap}, \mathrm{n}}+\mathrm{p} \boldsymbol{v}_{\mathrm{pp}, \mathrm{n})}\right.
$$

Where $v d r n, v d r$ are the drift velocity of electrons and holes, respectively. From this relation and the generalized Ohm's law, taking into account the formula $\mathrm{n} \cdot \mathrm{p}=\mathrm{n}$, it follows that the electrical conductivity of semiconductors can be written in the following form:

$$
\boldsymbol{\sigma}=\mathrm{q}\left(\mathrm{n} \boldsymbol{\mu}_{\mathrm{n}}+\mathrm{p} \boldsymbol{\mu}_{\mathrm{n}}\right)(\mathbf{5})
$$

Knowing the temperature characteristics of the concentration and mobility of free carriers, the experimental temperature dependence can be explained. For n-type impurity semiconductors based on $\mathrm{Ge}$ and $\mathrm{Si}$, these dependences are shown in Fig. 3. [2.26]
At temperatures $\mathrm{T}>\mathrm{Tmax}$, the electrical conductivity is determined by the product of the concentration and mobility of the native carriers, and with increasing $\mathrm{T}$, the carrier mobility decreases, and the concentration increases exponentially. The decrease in mobility $(\mathrm{T})$ in this region of temperature change is much weaker than the increase in the concentration $\mathrm{n}(\mathrm{T})$. As a result, at $\mathrm{T}>\operatorname{Tmax}$, the semiconductor's electrical conductivity increases with increasing temperature, and on a semi-logarithmic scale the function $=\mathrm{f}(1 / \mathrm{T})$ is almost linear with the slope tangent $\tan \sim \mathrm{E} 3$. At temperatures $\mathrm{T}<\mathrm{Tmax}$, the concentration of intrinsic carriers is low ( $p i<<$ $\mathrm{Nd}, \mathrm{Na}$ ) and the conductivity is mainly determined by the concentration of impurity carriers.

So, for example, in a donor semiconductor at temperatures characteristic of the area of operation of most semiconductor devices, when all impurity atoms are ionized, the electrical conductivity is

$$
\sigma=q n \mu_{n}=\mathrm{qN}_{\mathrm{A}} \mu_{n} .
$$

\section{CONCLUSION}

In this section of the change in the function $=\mathrm{f}(1 / \mathrm{T})$ (see Fig. 3), a slight decrease in the electrical conductivity of impurity semiconductors with increasing temperature is associated only with a decrease in the carrier mobility at their almost constant concentration.

In $G e$, the dependence of carrier mobility on temperature is weaker than in $\mathrm{Si}$, and the value in $G e$ is greater than in $\mathrm{Si}$. This is illustrated by fig. 3, 
where the dependences $=\mathrm{f}(1 / \mathrm{T})$ are shown for two different concentrations of $N D$ impurity in $S i$ and $G e$.

\section{List of main used is marked}

$\boldsymbol{\sigma}$ - Electrical conductivity

p- Resistivity

$\Delta E_{3 .-}$ semiconductor band gap

velocity

$\boldsymbol{V}_{\text {other- }}$ drift velocity and saturation drift

$\boldsymbol{\mu}$ - Carrier mobility

$\mathbf{N}_{A}, \mathbf{N}_{\mathrm{a}}$ atom concentration of donors and acceptors

$\mathbf{j}_{\mathrm{Ap}}$ - electron and hole current density

$E$ - electric field strength

$\boldsymbol{\mu}_{\mathrm{n}}, \boldsymbol{\mu}_{\mathrm{p}}$ - carriers

\section{REFERENCES}

1. A.Teshaboev, S. Zaynobiddinov, Sh.Ermatov. Solid state physics. Tashkent - "Finance" 2001.p.262

2. Г.Г.Шишкин., А.Г.иишкин. Элекироника. Москва-"Дрофа" 2009. 